\title{
Magnetic field camera for fast - high resolution inline magnet inspection
}

\author{
Dr. Koen Vervaeke \\ MagCam NV, Research Park Haasrode, Romeinse straat 18, B-3001 Leuven, Belgium \\ Tel.: +32 4945894 04, E-Mail: koen.vervaeke@magcam.com
}

\begin{abstract}
1. Introduction
Fast and accurate quality inspection of permanent magnets is increasingly important in development, production and quality control of ever more accurate and reliable sensors as widely used in automotive applications, more efficient electric drives for e.g. electric vehicles, new medical devices, consumer electronics and many other magnet applications [1-6]. Furthermore, in many high-end applications a 100\% magnet quality control is desired, but not feasible. Also in sensor R\&D there is a need for fast and advanced magnet characterization tools for developing nextgeneration sensor concepts and assemblies [3,4].

Different magnet types and applications require different parameters to be measured and compared to supplier spec sheets for quality control. For uniaxial magnets, the magnetization vector and its deviation angle are mostly important, while for multipole magnets the pole uniformity, north-south pole symmetry and deviations in pole segment angles are quality-defining parameters [5,6]. Also field homogeneity and material defects, as well as magnetization defects are important in many applications. Traditional magnetic measurement techniques are not capable of measuring all of these magnet characteristics in an economic way if at all. They have limited capabilities when it comes to their applicability for today's high demands in magnet quality control, either because they only measure one particular magnet property, are not suited for small magnets, have limited speed or are not suitable for automation (e.g. are not digital) or a combination of the above.
\end{abstract}

Driven by a clear industrial need for advanced inspection equipment for permanent magnets as outlined above, we recently reported on a new magnetic measurement technology, called a 'magnetic field camera' or 'MagCam', a powerful and unique measurement platform for fast and accurate live inspection of both uniaxial and multipole permanent magnets [7-9]. Its applications include manual and automated magnet inspection and characterization, as well as research and development of magnets and magnetic systems in a wide array of applications involving permanent magnets. This unique measurement instrument offers unprecedented characterization capabilities for various magnet and magnetic assembly properties. For uniaxial magnets the system measures the $x, y, z$-components of the magnetization vector and directly gives the deviation angle with respect to the geometrical symmetry axis. For multipole magnets an analysis is performed including pole height uniformity, high resolution zero-crossing distance/angle measurements, magnetic asymmetries and more. For magnets where field homogeneity is important, a homogeneity analysis can be performed in real time, identifying field inhomogeneities which are generally due to magnetic or mechanical defects in the magnet.

Further advancements in this technique are reported here, including advanced data analysis software algorithms which are able to extract unprecedented magnet property and quality information from the measured magnetic field maps, as well as the ability to implement the MagCam technique in automated production lines for fast - high resolution inline magnet inspection.

\section{MagCam measurement principle}

The MagCam measurement principle is based on a high resolution and high speed quantitative 2D mapping of the magnetic field distribution of a magnet, using a patented sensor chip with an integrated 2D array of $128 \times 128$ (= 16384) microscopic Hall sensors. The sensors have a pitch (spatial resolution) of $0.1 \mathrm{~mm}$ in both $\mathrm{X}$ and $\mathrm{Y}$ directions. Each Hall sensor has an active area of 40x40 micrometer and locally measures the magnetic field (i.e. the perpendicular component of it). All sensors are electronically scanned at high speed, resulting in a quantitative high resolution magnetic field map over an area of $13 \mathrm{~mm} \times 13 \mathrm{~mm}$. A full resolution MagCam magnetic field map is captured in less than 1 second. Figure 1 shows a photograph of the MagCam sensor chip, along with a typical measurement configuration and measured magnetic field distribution image, which is readily analyzed using various techniques, such as cross section analysis and statistical analysis. 

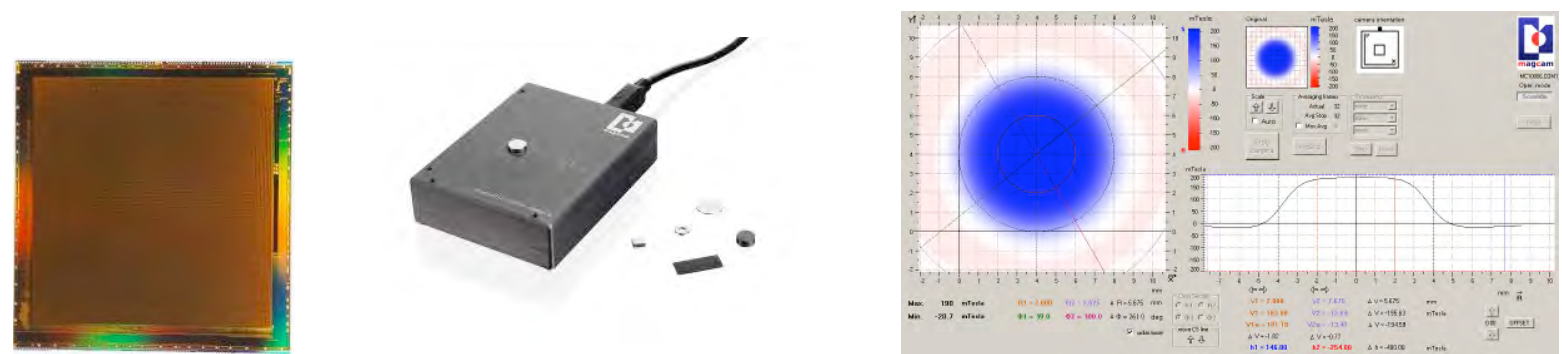

Figure 1: Left: MagCam sensor chip with an integrated 2D array of $128 \times 128$ microscopic Hall sensors.

Middle: cylindrical sensor magnet ( $8 \mathrm{~mm}$ diam., $2 \mathrm{~mm}$ height) on the sensitive surface of the MagCam sensor module. The magnet is magnetized along the axial direction.

Right: Software screenshot showing quantitative color plot of the measured magnetic field map (Bz component), along with real time cross section analysis and statistical analysis of the image.

The complete measurement setup consists of a magnetic field camera sensor hardware module and measurement and analysis software, capable of extracting unprecedented information from the measured magnetic field images. A compact setup is shown in Figure 2, where the sensor is connected to a computer. The measurement sample (usually a magnet) is placed on the sensitive sensor surface of the magnetic field camera. The magnetic field camera sensor measures high resolution 'images' of the magnetic field distribution of the magnet at high speed. The MagCam sensor is connected to a computer via a USB-cable, where the MagScope software runs measures and analyzes the magnetic field maps in real time.

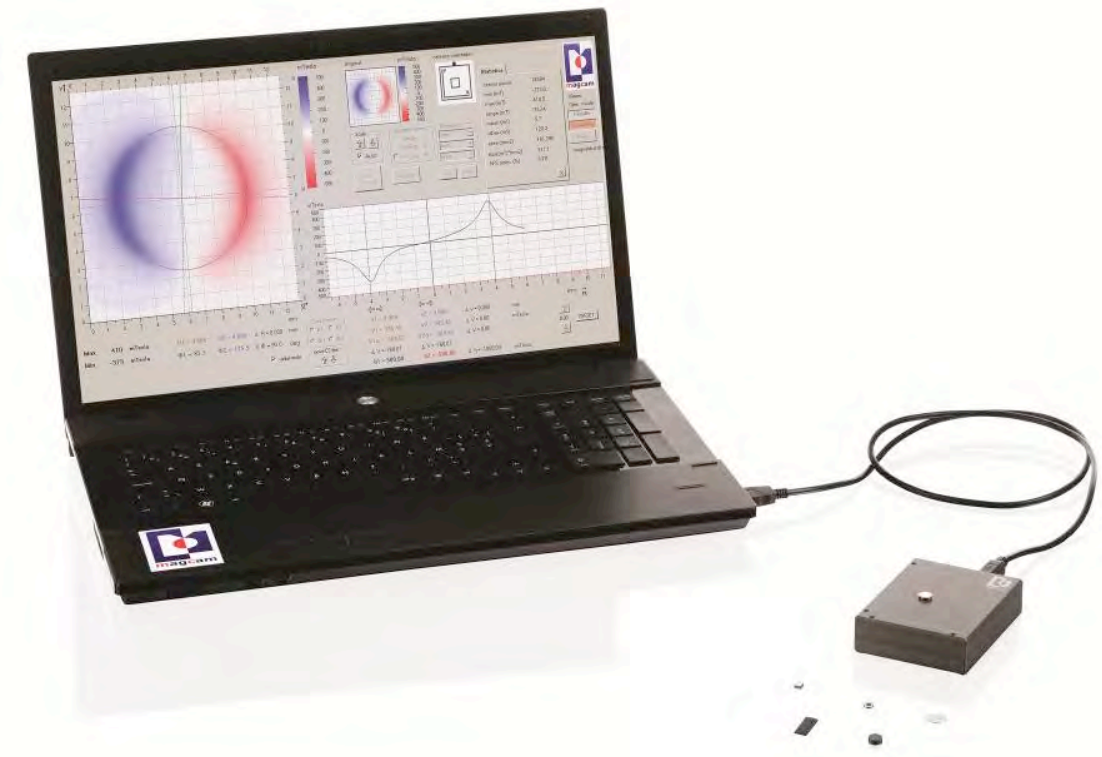

Figure 2: Measurement setup with MagCam magnetic field camera sensor module and MagScope software. The measured magnet is in this case a diametrically magnetized cylinder.

The system also allows for absolute magnet positioning, such that the physical magnet position is accurately known relative to the MagCam sensor chip and hence to the measured magnetic field map. This is done using magnet positioning frames, i.e. calibrated frames that can be easily mounted on the MagCam Sensor Module. When a magnet is placed against the frame, its exact position is known in the MagScope software, as shown in Figure 3. 

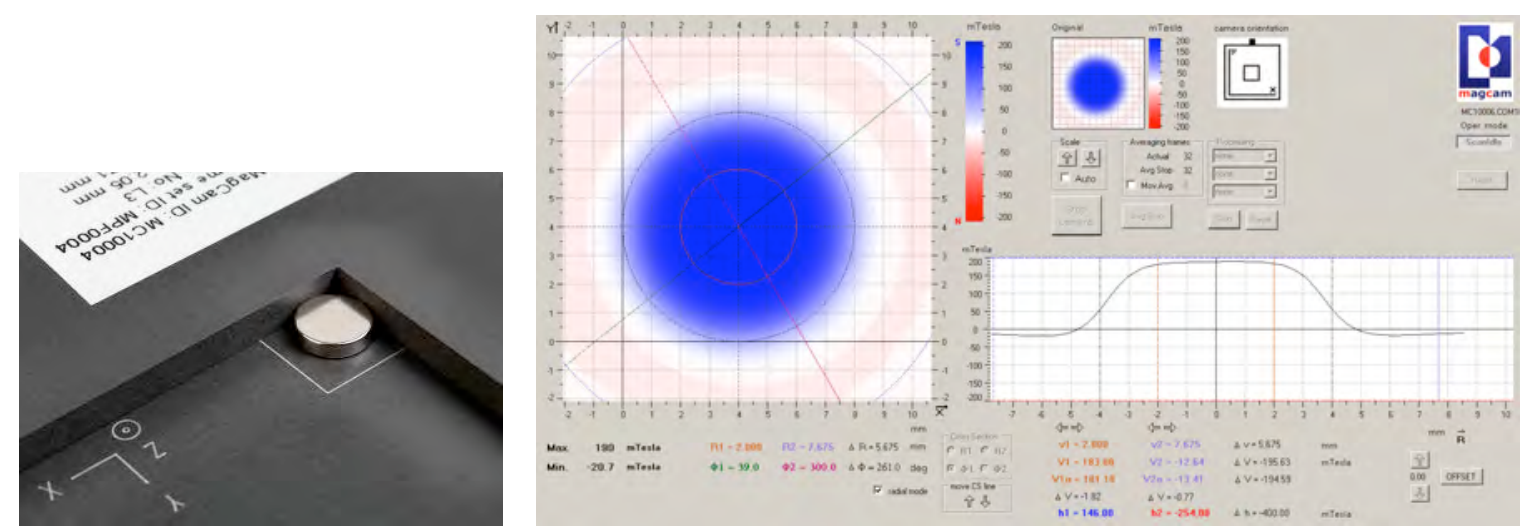

Figure 3: Left: a calibrated magnet positioning frame is used to accurately know the geometrical magnet position relative to the measured magnetic field map. Right: the analysis software displays the position of the frame and of the magnet and allows to perform e.g. analyses using a cylindrical coordinate system which is precisely centered in the geometrical magnet center.

\section{Data analysis of the magnetic field distribution}

The MagScope measurement and analysis software records and analyzes in real time the magnetic field maps measured from the MagCam sensor. The quantitative measured MagCam maps can be represented in various ways, such as using high resolution interpolated quantitative color scale graphs, as shown in Figure 4 . These graphs can be analyzed using both Cartesian and cylindrical coordinates, depending on the magnet geometry. In Cartesian coordinates, cross-section analyses can be performed across horizontal and vertical lines. In cylindrical coordinates, cross-section analyses can be performed across radial and circumferential lines. Along the cross-section lines, very accurate distance and angle measurements can be made between points of interest in the magnetic field map (e.g. magnetic field zero-crossings, poles, localization of specific magnetic field values, anomalies etc.). These analyses can be performed with absolute magnet position indication, when a positioning frame is used, as discussed above. The software can also perform image processing algorithms and statistical image analysis in real time.

These features allow to directly characterize a number of magnet properties such as multipole magnet angle failure, north-south pole asymmetry, magnet (in)homogeneity and material/magnetization defects. Also, quantitative measurements can directly be made of distances between poles and zeros, pole segment angles on multipole cylindrical and ring magnets, magnetic field distribution on lines and circles etc.

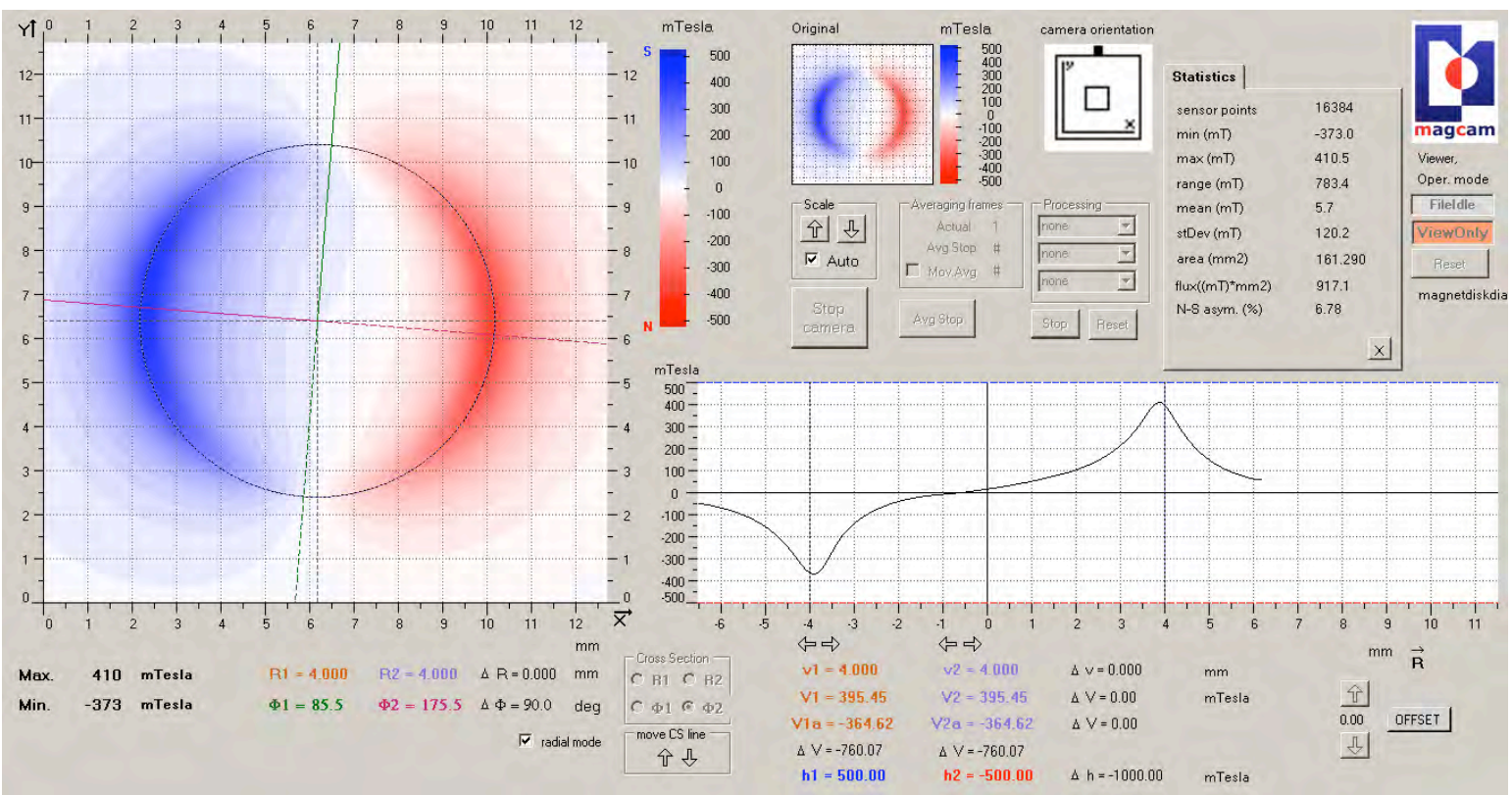

Figure 4: Screenshot of the MagScope measurement and analysis software (measurement data is from the diametrically magnetized magnet of Figure 2. Left is the quantitative interpolated color scale graph $(0.025 \mathrm{~mm}$ pixel resolution) and right the cross-section graph along the purple diametrical line on the left. The circle in the color graph is positioned exactly on the geometrical magnet circumference, allowing to analyze the magnetic field distribution relative to the geometrical magnet position. (In this case the magnet position was not determined using a positioning frame, but resulted from a MagFit analysis, which also yields this result, as discussed furhter.) In the upper right area the image statistics are displayed. The complete analysis can be performed in real time during measurement. 
Even more information can be extracted from the MagCam maps using the in-house developed 'MagFit' Magnet Analysis Software, which offers advanced MagCam data analysis capabilities for a complete characterization of uniaxial permanent magnets. Based on theoretical magnet models, MagFit calculates a best fit to the measured MagCam map by optimizing a set of model parameters and constructs a theoretical magnet model that best approximates the measured MagCam map. The magnet model parameters that are optimized in this procedure provide direct quantitative values for the magnet's properties and thus a lot of extra information is extracted from the measurement data, including the full remanence magnetization vector and its angle deviation, deviations from a 'perfect' magnet, the full B-vector field in 3D space, local defects in the magnetization or in the magnet material, the exact magnet position and orientation, all in a matter of seconds (see Figure 5). Since all resulting parameters are quantitative and relevant magnet characteristics, they can be used for e.g. a quantitative pass/fail quality control based on user-defined tolerances.

Other MagFit output includes the fitted (theoretical) MagCam map, the residual map (difference between fit and measurement) and the other components of the magnetic field (Bx and By). Examples are provided further on. MagFit uses a fast algorithm that allows to reach a solution in a matter of seconds (typically 2 seconds), which makes the analysis suitable for fast in-line applications.

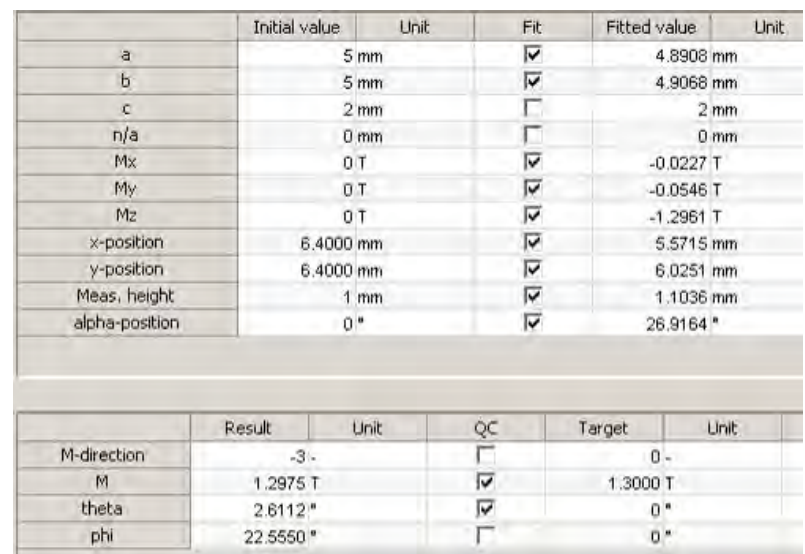

Figure 5: Screenshot of the MagFit magnet analysis software module, showing analysis results for a uniaxially magnetized block magnet (magnetized along the z-direction). The software optimizes a number of model parameters in order to obtain a best fit of a theoretical magnet model to the measured MagCam data. Among the output parameters are the full remanence magnetization vector and its deviation angle from the magnet's main geometrical axis, the exact magnet position and orientation, and the magnet sizes. Other output includes the fitted (theoretical) MagCam map.

\section{Industrial automation setup}

The complete functionality of the MagCam system can be automated, including the advanced data analysis capabilities such as MagFit. This is possible via an in-house developed C-language API (Application Programming Interface), which allows users to integrate complete MagCam measurement and analysis cycles into other computer programs processes. The MagCam system can thus be implemented in e.g. automated production lines. The inherent speed of both measurement and analysis opens new opportunities for e.g. a $100 \%$ inline magnet inspection. Other possibilities include mounting a MagCam sensor onto an industrial robot to scan the magnetic field distribution of more complex structures, such as e.g. larger rotors containing multiple permanent magnets.

\section{Application examples}

Using the measurement setup described above, a number of typical measurements and analysis examples are presented below, performed on different types of commonly used sensor magnets, including both multipole and uniaxially magnetized magnets.

\section{Multipole ring magnet inspection}

Permanent magnets with multipole magnetization patterns are widely used in sensor applications, magnetic couplings and small electric motors. Up to now, no economic measurement equipment was available to thoroughly inspect the magnetic quality of multipole magnets. The MagCam platform opens new possibilities to perform fast and detailed inspection of multipole magnets, giving access to a number of important magnet characteristics which are deterministic for the magnet's quality.

Figure 6 shows a ring magnet with a 4-pole axial magnetization profile. This type of magnets finds applications in e.g. magnetic couplings. 

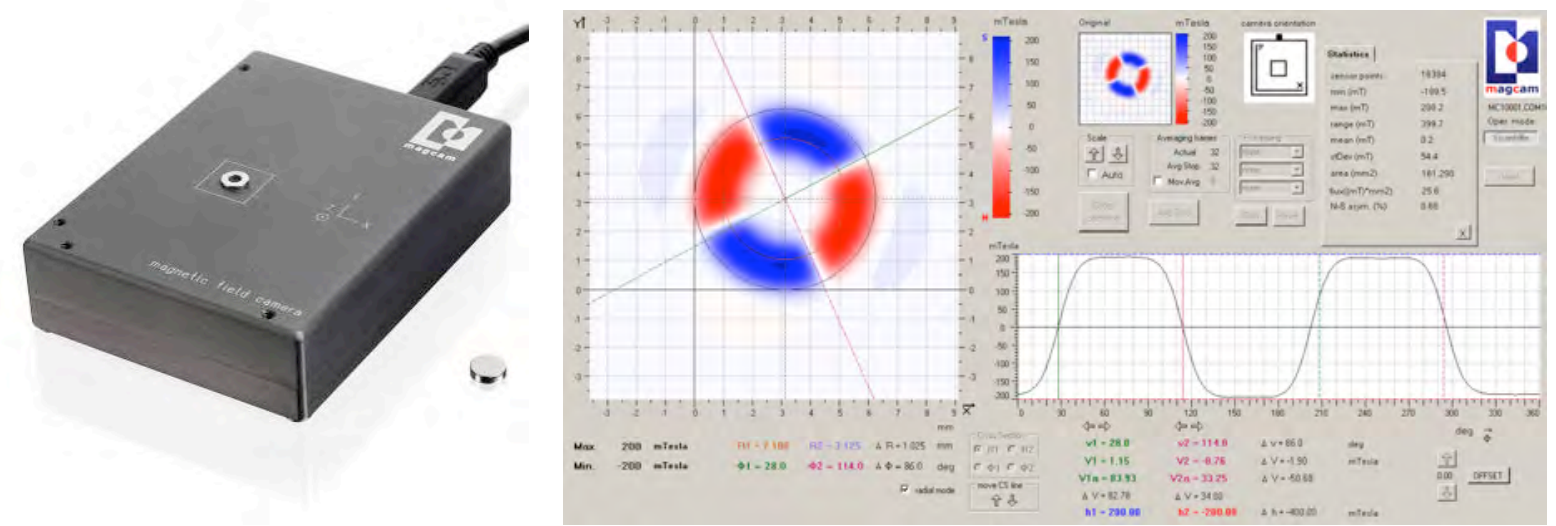

Figure 6: Left: Ring magnet with 4-pole axial magnetization pattern placed on the MagCam sensor surface Right: MagCam map of the ring magnet shown on the left. The actual measurement was performed using a magnet positioning frame such as shown in Figure 3 (not shown in this figure). The frame's position is shown in the color graph, together with the magnet's geometrical position (circumference and center). The magnetic field map is analyzed using cylindrical coordinates. The cross-section view on the right is taken on a circle with radius $R=2.1 \mathrm{~mm}$. On this cross-section the size of each pole segment can be very accurately measured.

Three important parameters define the quality of the magnet in Figure 6:

- The absolute value of the magnetic field;

- The pole segment angle uniformity, determined by the border between north and south pole segments;

- The uniformity and homogeneity of the magnetic field across the different poles.

All three of these parameters can be measured in real time using the MagCam system.

The first parameter, the absolute value of the magnetic field, is readily available from the calculated statistics from the MagCam map (see right side in Figure 6). Several measures for this quantity can be used, such as the maximum and minimum magnetic field values $(+200 \mathrm{mT}$ and $-200 \mathrm{mT}$ in this case). Another measure is the average absolute magnetic field, which is calculated as the mean value of the absolute value of all pixels in the MagCam map. In this case, this value is $23.2 \mathrm{mT}$. The advantage of this quantity is that it takes into account the complete magnetic field distribution and not only two single points such as the maximum and minimum values represent.

The second quality-parameter, the pole segment angle uniformity, can also be directly measured from the MagCam map, as illustrated below. In the present measurement, a magnet positioning frame was used to know the exact magnet position and hence the geometrical center of the magnet, which is used as the origin of the cylindrical coordinate system. In automated setups, the accurate positioning can be performed by e.g. a robot. A circumferential cross-section is taken along the R1 (orange) circle in the color graph in Figure 6 . This cross-section is represented in the graph at the right. On this cross-section graph, angles can be accurately and quantitatively measured. Using the two radial lines phi1 (green) and phi2 (purple), the absolute angular size of each pole segment is measured by taking the angular difference between the zero-crossing points in the graph. For a perfect 4-pole magnet, each segment should measure exactly $90^{\circ}$. For this particular magnet, the measured segment angles are shown in Table 1.

Table 1: Pole segment angles measured from the MagCam map in Figure 6.

\begin{tabular}{ccc}
\hline \hline Pole no. & $\begin{array}{c}\text { Segment angle } \\
\left({ }^{\circ}\right)\end{array}$ & Deviation from $90^{\circ}\left({ }^{\circ}\right)$ \\
\hline S1 & 86.0 & -4.0 \\
N1 & 88.5 & -1.5 \\
S2 & 93.5 & 3.5 \\
N2 & 92.0 & 2.0
\end{tabular}

Pole numbers are counted counterclockwise, starting at the top south pole in Figure 6.

From the values in Table 1 we see that the consecutive segments $\mathrm{S} 1$ and $\mathrm{N} 1$ are smaller than $90^{\circ}$, and that $\mathrm{S} 2$ and $\mathrm{N} 2$ are larger than $90^{\circ}$. An explanation for this deviation is that during magnetization, the magnet was not perfectly aligned with the magnetization yoke.

Segment angle deviations can cause asymmetric coupling performance and hence unwanted vibrations, loss of accuracy and other effects, when used e.g. in a magnetic coupling application. When they are used in sensor applications, they directly convey the pole segment angle deviations to the sensor output signal. With the MagCam system, it is now possible to quantitatively measure this parameter and perform quality control before the magnet is built into an assembly. 
The third quality parameter, pole height uniformity, is also readily analyzed using the MagScope software, as shown in Figure 7.

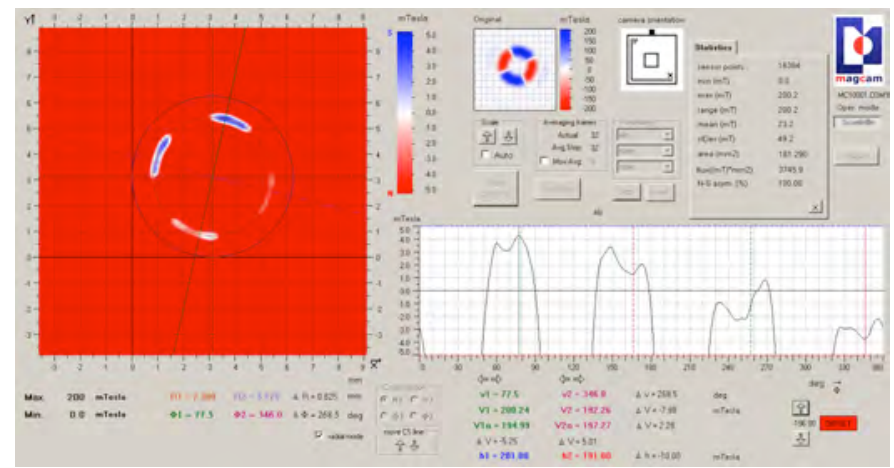

Figure 7: Same MagCam map as in Figure 6, but with some image processing applied. First, all magnetic field values are made positive (absolute value). Subsequently a uniform offset is applied to the data, as to be able to zoom into the pole region. This way all poles (north and south) can be compared to each other simultaneously and pole height uniformity, north-south asymmetry and detailed pole shape can be directly and quantitatively examined.

In order to analyze the peak uniformity of all poles simultaneously (both north and south), data processing is applied to the MagCam image. First all magnetic field values are made positive (absolute value). Subsequently a uniform offset is applied to the data, as to be able to zoom into the pole region. This way all poles (north and south) can be compared to each other simultaneously and pole height uniformity, north-south asymmetry and detailed pole shape can be directly and quantitatively examined. A reference image showing the original MagCam map (top center in Figure 7) allows to retrieve which poles are north and which are south. From the cross-section view along the circumferential line at a radius $\mathrm{R} 1=2.3 \mathrm{~mm}$ (this is the radius which maximizes the pole values) a pole height difference of about $8 \mathrm{mT}$ ( $=4 \%$ of pole height value) is observed (see Figure 7). Poles S2 and N2 are observed to be significantly weaker than S1 and N1. The weaker poles correspond to the poles with the larger segment values (see Table 1). Whether these two quantities (pole height and segment angle) are correlated would be subject of further and more systematic investigation.

It is however clear that such asymmetry in the pole height would directly translate in an asymmetry in the coupling force when such magnet is built into a magnetic coupling, which in turn can cause vibration and hence accuracy and efficiency loss.

The above example shows how multipole magnets can be inspected in a very detailed and economical way using the magnetic field camera principle.

\section{Uniaxial magnet inspection}

Uniaxially magnetized magnets are the most common form of permanent magnets and are also widely used in sensor applications. Typical magnet materials include SmCo, Ferrite, AINiCo and NdFeB. A uniaxial magnet typically has a basic geometrical shape (block, disk, ring, segment) with one magnetization axis which is usually along one of the main geometrical axes or perpendicular to it. Especially $\mathrm{NdFeB}$ magnets suffer from a deviation of the magnetization axis relative to the geometrical magnet axis. This is due to the crystalline nature of the material, which crystal axes are not always parallel to the geometrical axes of the machined magnet. This deviation can be characterized by the angle the magnetization vector makes with the geometrical main axis. This 'angle deviation' or 'skew angle' directly translates into measurement deviations when the magnet is built into a sensor assembly. The angle deviation is therefore one of the most important characteristics of a uniaxial sensor magnet and should be as small as possible (ideally $0^{\circ}$ ). For small magnets however, traditional magnetization measurement equipment, such as Helmholtz coils, become inaccurate due to the small amount of material in the magnet. Moreover, Helmholtz coils only measure one parameter, i.e. the global mean remanence magnetization vector of the magnet, without any local information (i.e. homogeneity information).

The measurements and analysis in this section show that the MagCam setup is able to measure the full magnetization vector and its deviation angle. On top of that, also the magnet's size, position and orientation are obtained. This is done using the advanced MagFit analysis algorithm that fits a theoretical magnet model to the measured MagCam data and in the process optimizes a number of model parameters to obtain a best fit theoretical MagCam map. This theoretical MagCam map is also available as output from the analysis, among much more. Moreover, when subtracting the theoretical map from the measured map, a 'residual' map is obtained, which shows all deviations of the measured MagCam map from the theoretical best fit map. On this residual map local deviations from the 'perfect' magnet are very clearly visible. These deviations can be due to material defects such as impurities or structural defects in the magnet material, or defects in the magnetization of the magnet. This residual map is perfectly suited to run a statistics analysis, yielding quantitative characteristics of the deviation of the magnet from a perfect magnet. These image statistics can in turn be used to be tested against user-defined tolerance limits for e.g. a pass/fail quality control. 


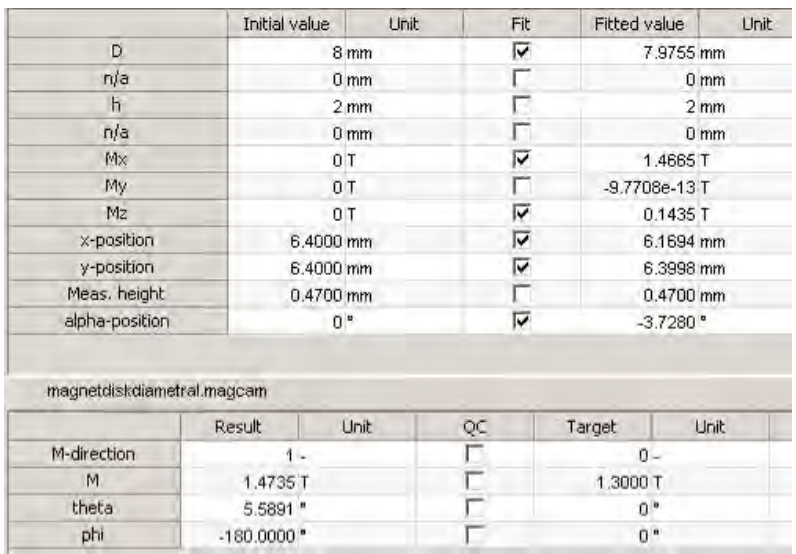

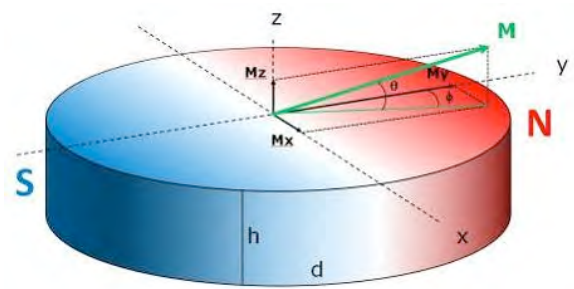

Figure 8: Left: Screenshot of the MagFit magnet analysis software, showing analysis results for the diametrically magnetized cylindrical magnet from Figure 4. The software optimizes a number of parameters in order to obtain a best fit of a theoretical magnet model to the measured MagCam data. Among the output parameters are the full magnetization vector (with size $=1.38 \mathrm{~T}$ ) and its deviation angle from the magnet's main geometrical axis (in this case theta $=5.6^{\circ}$ ), the exact magnet position and orientation, and the magnet sizes. Other output includes the fitted (theoretical) MagCam map, the residual Bz-map and the Bx and By maps.

Right: 3D-sketch of the magnet under test with indication of some parameters that result from the MagFit analysis, such as the full magnetization vector and its deviation angle from the main (geometrical) axis, as well as the position of the north and south poles.

Once MagFit has optimized the parameters for the theoretical magnet model, the other components of the magnetic field distribution (Bx and By) can also be computed, as shown in the figures below.
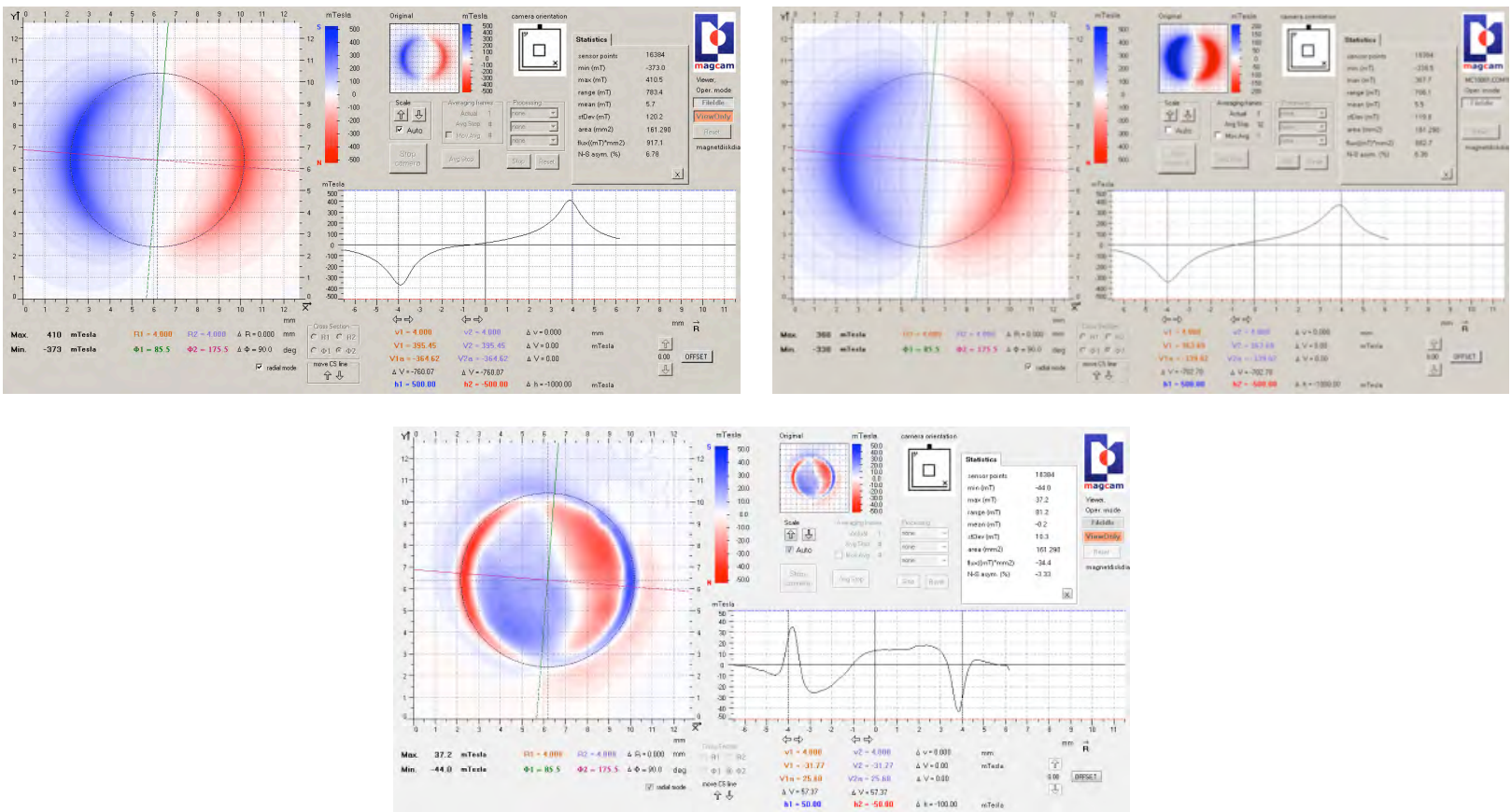

Figure 9: Top left: measured MagCam image;

Top right: Fitted Bz component of the magnetic field distribution, resulting from the MagFit analysis.

Bottom: Residual image (difference between measurement and theoretical best fit images). The residual image shows all deviations from the 'perfect' theoretical magnet, such as magnetization inhomogeneities and material defects. Note the scale difference of the measured/theoretical images (+/- 500mT) and of the residual image (+/$50 m T)$. 

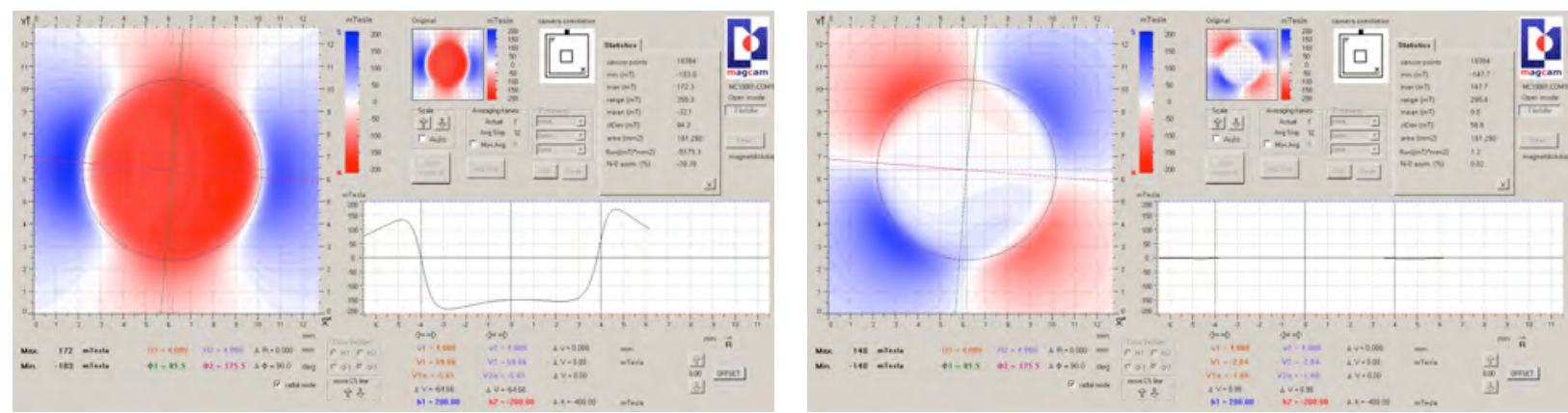

Figure 10: Bx component (left) and By component (right) of the magnetic field distribution, as calculated from the optimized best fit theoretical magnet model. Note the influence of the magnetization vector skewness, which is manifested in an asymmetry of the magnetic field distribution.

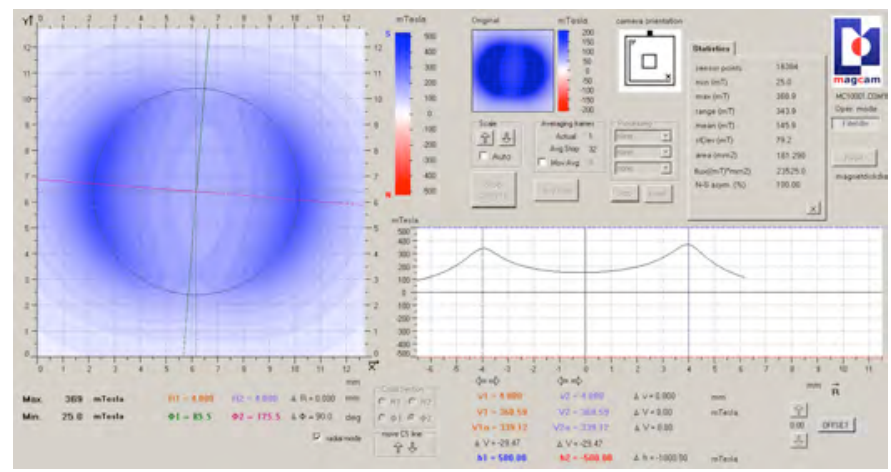

Figure 11: Absolute value of the magnetic field distribution $=\operatorname{sqrt}\left(B_{x}{ }^{2}+B_{y}{ }^{2}+B_{z}{ }^{2}\right)$.

\section{Conclusion}

Driven by an industrial need for advanced inspection equipment for permanent magnets in many different applications, among which the high-quality magnets used in sensor applications, we have presented the capabilties of the magnetic field camera (MagCam) measurement and analysis system, a powerful and unique measurement platform for fast and accurate live inspection of both uniaxial and multipole permanent magnets. Measurements and analyses on different kinds of magnets were performed, showing the capabilities of the system to perform a full characterization of small permanent magnets, including the quality-defining characteristics such as angle failure, pole symmetry and homogeneity for multipole magnets and magnetization vector size and angle deviation for uniaxial magnets.

Using advanced data analysis and combining theoretical magnet modeling and MagCam measurements, a lot of extra information can be extracted from the measurement data, including the magnet's exact position and orientation, the magnetization vector, a theoretical best fit to the MagCam map and a residual MagCam map showing all 'imperfections' in the magnet.

This powerful magnet inspection platform is well suited for both R\&D and industrial (automated) magnet inspection, since it is completely digital, gives fast measurement and analysis results (in seconds), and can be integrated in production lines. These unique characteristics make that the MagCam system is quickly becoming the new industry standard for magnet inspection in virtually all industries where high quality permanent magnets are used.

\section{References}

[1] www.melexis.com , Magnet Selection Application Note MLX90316

[2] V Sankaranarayanan, Magnetic Sensors Expand their Reach from Automotive to Cell Phones and Consumer Electronics Applications, Frost \& Sullivan Market Research Report (2010)

[3] U. Ausserlechner, Inaccuracies of Giant Magneto-Resistive Angle Sensors Due to Assembly Tolerances, IEEE Trans. Magn. 45, No 5, 2165 (2009)

[4] www.arnoldmagnetics.com , Technical papers and presentations, Polymer Bonded Magnets

[5] Y.N. Zhilichev, Precise Multipole Magnetization of Disk Magnet for Sensor Applications, IEEE Trans. Magn. 39, No 5, 3301 (2003)

[6] J. Paulides et al., Sinusoidal Behavior of a Dipole Magnetization for Position Sensing Applications, IEEE Trans. Magn., Vol. 42, No. 10, 3294 (2006)

[7] www.magcam.com , Technology page

[8] K. Vervaeke, Microscopic Hall sensors for Magnetometry and Local Magnetic Imaging, Ph.D. Thesis at the University of Leuven, Belgium (2007)

[9] Conference Proceedings of EDPC 2011 Conference (27-30 September 2011, Nürnberg), ISBN 978-1-4577-13682 (seite 233) 